\title{
A PESCA REALIZADA NAS COMUNIDADES DE PESCADORES EM REPRESAS DO ALTO TIETÊ, SÃO PAULO, BRASIL
}

CINTIA ALVES PARRAS ${ }^{1}$

WELBER SENTEIO SMITH²

1. Graduada em Ciências Biológicas, Universidade Cruzeiro do Sul, cintiaparras @yahoo.com.br

2. Professor Doutor, Programa de Mestrado em Processos Tecnológicos e Ambientais, Universidade de Sorocaba (UNISO), Laboratório de Ecologia Estrutural e Funcional, Universidade Paulista (UNIP), welber_smith@uol.com.br

*AUTOR CORRESPONDENTE: Prof. Dr. Welber Senteio Smith

Endereço: Rod. Raposo Tavares km 92,5 CEP 18023-000, Sorocaba, SP, Brasil

Email: welber_smith@uol.com.br

Recebido em: 30/05/2014 - Aprovado em: 30/06/2014 - Disponibilizado em: 30/07/2014

RESUMO: O Rio Tietê sofre grandes alterações ao longo do seu curso e uma alteração muito importante é a construção de reservatórios, que causam modificações na estrutura da comunidade de peixes. Essas alterações se dão na abundância e riqueza das espécies, na dieta, reprodução e na movimentação das espécies migradoras. A pesca é uma das atividades humanas mais importantes, constituindo-se em fonte de alimento, comércio, renda e lazer para grande parte da população, especialmente a que reside nas margens dos rios e represas. Este trabalho teve como objetivo caracterizar a atividade de pesca desenvolvida nos reservatórios Jundiaí e Taiaçupeba, localizados no Alto Tietê. Foram entrevistados 40 pescadores, com idade entre 33 e 69 anos de idade, sendo que destes, 10 são profissionais e 30 esportivos, que pescam nos finais de semana como lazer e todo pescado é usado para consumo próprio.

PALAVRAS-CHAVE: pesca artesanal, pesca esportiva, reservatório, Alto rio Tietê

\section{THE FISHERY IN THE FISHERMEN COMMUNITY IN RESERVOIRS OF THE ALTO TIETE RIVER, SÃO PAULO, BRAZIL}

\begin{abstract}
Tietê river is under great stress along its course and reservoir building is a major factor which alters the community's fish structure. Those alterations reflect in the abundance and richness, in the fish diet, reproduction and in the movement of migratory species. Fishing is one of the more important human activities, providing in food, trade, income and leisure to people, especially the ones that resides at the margins of rivers and reservoirs. This paper characterizes the fishing activity carried out by sporting and professional fishers in the Jundiaí and Taiaçupeba in Alto Tietê. Through interviews, we collected information about the capture methods, fishing spots, sazonality and captured fish species. We interviewed 40 fishers with age between 33 and 69 years, and of these, 10 professional and 30 sporting that fish in the weekends as leisure and every fish is used for own consumption.

KEYWORDS: reservoir, small-scale fishery, sporting fishery, High Tietê River.
\end{abstract}




\section{INTRODUÇÃO}

No Brasil, empreendimentos como as construções de reservatórios, na maioria das vezes, visam a produção de energia elétrica ou o abastecimento de água (BRITSKI, 1994). Além disso, é utilizada para irrigação, cultivo de organismos aquáticos, navegação, recreação e pesca (SMITH, 2003).

A inserção das barragens e a criação de lagos artificiais, acarretam um complexo de impactos que afetam os componentes químicos, físicos e biológicos, originalmente presentes no ambiente (AGOSTINHO, 1994). Além disso, altera o fluxo de água, modifica a estrutura do ecossistema, promove alterações ambientais, como modificações nos cursos d'água, e acarreta mudanças nas características naturais das comunidades de peixes (SMITH e BARRELLA, 2000; DELARIVA, 2002).

Algumas espécies de peixes de água doce são muito restritas em certos tipos de habitat, e em seus hábitos alimentares, pelas características e capacidade de suporte do ecossistema que habitam (HARDOY et al., 1992). A alimentação dessas espécies está relacionada ao tipo de ambiente em que vivem e a abundância de alimento, que o ambiente proporciona. Assim, como a comunidade de peixes presente em um reservatório é resultante da colonização feita por aquela anteriormente presente no rio, espécies que apresentam maior capacidade de adaptação ao novo ambiente proliferam, enquanto outras, podem ter sua abundância reduzida, ou mesmo, serem eliminadas (AGOSTINHO et al., 1992).

No Alto Tietê, devido a redução das espécies reofílicas e daquelas que tiveram sua população reduzida por não terem se adaptado ao novo ambiente predominantemente lêntico, foram introduzidas espécies exóticas, de maior valor econômico, como a tilápia (Tilapia rendalli), a tilápia do Nilo (Oreochromis niloticus) e a carpa (Cyprinus carpio). Esta ação visou minimizar as perdas causadas pelo barramento do rio Tietê, que afetou as espécies migratórias. Essa ação teve como principal objetivo beneficiar as comunidades pesqueiras (PEREIRA et al., 2001), que utilizam o pescado como fonte de alimento, de renda e lazer (ROOSEVELT et al., 1991; MEGGERS, 1997).

A principal pesca realizada no Brasil é a artesanal, onde o pescador trabalha sozinho, ou utiliza mão-de-obra 
familiar, ou não assalariada, ou em parcerias onde captura espécies de peixes utilizando equipamentos relativamente simples. Os pescadores artesanais retiram da pesca sua principal fonte de renda, mesmo exercendo outras atividades complementares (DIEGUES, 1988).

É através dessa pesca que adquirem um vasto conhecimento sobre o ambiente, o manejo dos instrumentos de pesca, biologia de certas espécies de peixes, como hábito alimentar, comportamento, reprodução e classificação, os tipos de ambientes propícios à vida dos peixes e identificação dos pesqueiros (melhores pontos de pesca) (DIEGUES, 1995; SILVANO, 1997).

No Brasil, a pesca artesanal, mesmo sendo difusa e de difícil controle (BAYLEY e PETRERE, 1989), é fundamental na produção pesqueira, sendo responsável por mais de $50 \%$ dos desembarques (CETRA e PETRERE, 2001). Nos rios e represas do Estado de São Paulo, e em várias regiões do Brasil, a pesca é caracterizada por não apresentar pontos fixos de desembarques, o pescador procura um local que seja mais produtivo (CETRA e PETRERE, 2001). Só no Estado de São Paulo é realizada em sete regiões: região do Rio Paraná, do Rio Grande, do Rio Tietê, Rio Paranapanema,
Rio Paraíba do Sul, dos Rios Mogi Guaçu e Pardo e do Rio Ribeira do Iguape, além do Complexo Billings (SANTOS et al., 1995; MINTE-VERA e PETRERE, 2000; CASTRO et al., 2003; CASTRO et al. 2006).

Portanto, a obtenção da máxima produtividade pesqueira e a preservação da ictiofauna dependem, principalmente, de um programa de administração pesqueira que inclua levantamentos limnológicos e ictiológicos (VIO, 1994) e que a ocorrência da introdução de espécies, visando organizar um polo de pesca esportiva, pode implicar numa perturbação do equilíbrio ambiental, gerando prejuízos do ponto de vista pesqueiro e ecológico (SMITH, 2006).

O presente trabalho teve como objetivo caracterizar a atividade pesqueira desenvolvida por pescadores esportivos e profissionais nos reservatórios Jundiaí e Taiaçupeba, localizados no Alto Tietê. Foram levantadas informações referentes aos métodos de captura, pontos de pesca, sazonalidade da pesca e espécies capturadas. Além disso, foram anotadas as condições ambientais que influenciam a atividade pesqueira, segundo o conhecimento dos pescadores esportivos e profissionais. 


\section{MATERIAIS E MÉTODOS}

O levantamento foi realizado nas Represas Jundiaí, em Mogi das Cruzes, e Taiaçupeba, na divisa de Mogi das Cruzes com Itaquaquecetuba (Figura 1), que constitui a Bacia Hidrográfica do Alto Tietê. A área de drenagem possui 1889 $\mathrm{km}^{2}$ e é constituída pelo rio Tietê (desde sua nascente até a divisa com Itaquaquecetuba). Estão presentes, também, os reservatórios Ponte Nova (no município de Salesópolis), Biritiba (em Biritiba-Mirim) e Paraitinga (em Salesópolis), tendo sido os dois últimos recentemente concluídos (REDE DE ÁGUAS, 2002).

Figura 1- Localização dos reservatórios utilizados no presente trabalho.

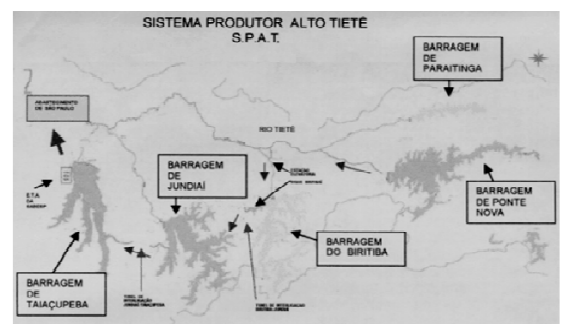

As represas Taiaçupeba e Jundiaí foram contruídas em 1976 e 1992, respectivamente. Elas formam o Sistema Produtor Alto Tietê, que constitui uma sucessão em cascata, na qual os reservatórios são interligados através de sistemas de túneis e canais, responsáveis pela diminuição dos poluentes ao longo da sistema, pois o reservatório a montante tem o papel de reter parte dos poluentes e nutrientes, levando a uma melhora na qualidade das águas e sedimentos ao longo da cascata de reservatórios (SISTEMA DE PRODUÇÃO DO ALTO TIETÊ, 1994; BARRELLA e PETRERE, 2003).

No período de agosto de 2006 a agosto de 2007, foram realizadas entrevistas, por meio de questionários, a dois tipos de pescadores: esportivos e profissionais. O levantamento foi realizado nas comunidades pesqueiras das Represas Jundiaí e Taiaçupeba.

No primeiro momento, foram preenchidas fichas de identificação, que constavam dados pessoais como: endereço, idade, grau de escolaridade, profisssão, cidade onde reside, se possui alguma propriedade e qual renda mensal. Além da frequência da prática de pesca, equipamentos e técnicas utilizadas para captura de peixes, qualidade do local freqüentado e as espécies de peixes mais procuradas.

O questionário para os pescadores esportivos visou buscar as seguintes informações: 1) tamanho mínimo para capturar os peixes; 2) distinção de um peixe jovem de um adulto; 3) as 
consequências de pescar peixes jovens; 4) a necessidade da informação a respeito do tamanho mínino dos peixes a serem pescados e como essa informação poderia ser transmitida.

O questionário para os pescadores profissionais objetivou levantar as seguintes informações: 1) forma de comercialização do peixe; 2) mês, estação do ano e hora do dia para melhor pescar; 3 ) qualidade do local de pesca; 4) área mais frequentada pelos pescadores esportivos; 5) relação turística $X$ pesca profissional.

Todo o questionário foi aplicado aleatoriamente, entre os pescadores que atuavam nas duas Represas. As entrevistas

\section{RESULTADOS}

Foram entrevistados, na represa Jundiaí e na represa Taiaçupeba, um total de 40 pescadores, sendo que destes, 30 esportivos e 10 profissionais. Com as informações obtidas nas entrevistas, podese traçar o perfil dos pescadores. A faixa etária dos pescadores esportivos variou de 34 e 63 anos, com uma média de 48,5 anos, com desvio padrão de 10,72 (Tabela 1); já os pescadores profissionais apresentaram média de 51 anos, com desvio padrão de 12,71, variando de 33 a 69 anos (Tabela 2). O sexo masculino com os pescadores esportivos foram feitas em pontos ao redor das represas, onde o acesso à água fosse facilitado para o uso de varas de pesca, e as entrevistas com os pescadores profissionais foram realizadas nos pontos de desembarques pesqueiros ou os pescadores eram encontrados desenvolvendo alguma outra atividade relacionada à pesca, como limpeza e conserto de redes e aparelhos. Durante as entrevistas, levantou-se as espécies de peixes capturadas. As informações obtidas nas entrevistas, relativas aos dados socioeconômicos e pesqueiros foram agrupadas e analisadas de forma qualitativa e quantitativa (TRIOLA, 2005).

apresentou-se dominante, as mulheres apenas faziam companhia e assistiam a pesca. Em relação à escolaridade, 100\% não concluíram o ensino fundamental.

Para os pescadores esportivos, a renda familiar não provem da pesca; os pescados são para consumo próprio ou da família, e a pescaria geralmente se dá nos finais de semana e feriados, como lazer, porque já têm outra atividade econômica como: pedreiro, pintor, funcionário público, ajudante geral, mecânico, caseiro, torneiro mecânico, motorista, segurança, 
auxiliar de pedreiro, além dos aposentados (Tabela 1). Todos os entrevistados possuem casa própria e pescam naquela região porque residem em bairros ou cidades próximas.

Tabela 1 - Informações dos pescadores esportivos das represas Jundiaí e Taiaçupeba.

\begin{tabular}{lll}
\hline & $\begin{array}{l}\text { Represa Jundiaí } \\
(\mathbf{n = 1 6})\end{array}$ & $\begin{array}{l}\text { Represa Taiaçupeba } \\
(\mathbf{n}=\mathbf{1 4})\end{array}$ \\
\hline Idade & $34-51$ anos & $36-63$ anos \\
Sexo & $100 \%$ masculino & $100 \%$ masculino \\
instrução & $100 \%$ ensino & $100 \%$ ensino \\
Outra atividade & fundamental & fundamental \\
& $32,3 \%$ aposentados & $28,6 \%$ aposentados \\
& $18,7 \%$ pedreiros & $28,6 \%$ pedreiros \\
& $12,5 \%$ ajudante & $14,4 \%$ ajudante geral \\
& geral & \\
& $12,5 \%$ caseiros & $7,1 \%$ motoristas \\
& $6, \%$ pintores & $7,1 \%$ caseiros \\
& $6 \%$ funcionário & $7,1 \%$ segurança \\
& público & \\
$6 \%$ mecânicos & $7,1 \%$ auxiliar de \\
& $6 \%$ torneiro & \\
& mecânico & \\
\hline
\end{tabular}

Tabela 2 - Informações dos pescadores profissionais das represas Jundiaí e Taiaçupeba.

\begin{tabular}{lll}
\hline & $\begin{array}{l}\text { Represa Jundiaí } \\
(\mathbf{n = 6})\end{array}$ & $\begin{array}{l}\text { Represa Taiaçupeba } \\
(\mathbf{n = 4})\end{array}$ \\
\hline Idade & $33-59$ anos & $44-69$ anos \\
Sexo & $100 \%$ masculino & $100 \%$ masculino \\
Grau & $100 \%$ ensino & $100 \%$ ensino \\
instrução & fundamental & fundamental \\
Outra atividade & $66,7 \%$ aposentados & $25 \%$ aposentados \\
exercida & & \\
\hline
\end{tabular}

Ao contrário, os pescadores quanto da represa Taiçupeba, constroem "edículas" de madeira com finalidade de abrigo e depósito dos equipamentos de pesca como barcos e redes. A pesca é praticada diariamente, com exceção dos finais de semana, quando os pescadores retornam para suas residências. Todo o pescado é responsável pela renda familiar, que pode variar, aumentando com a captura de espécies como a carpa $(C$. carpio) e as tilápias ( $T$. rendalli e $O$. niloticus), que são espécies que têm maior valor econômico quando comparadas com outras espécies. Os peixes são vendidos no atacado ou no varejo para a população local, bares e lanchonetes da região.

$\mathrm{O}$ método e o equipamento de pesca estão relacionados com o tipo de peixe que será capturado. Entre os equipamentos mais utilizados, estão as redes de espera, com malhas de diferentes tamanhos $(3,0 \mathrm{~cm}$ a $13,0 \mathrm{~cm}$, entre nós opostos ou adjacentes). Barcos motorizados ou a remo foram citados apenas pelos pescadores profissionais (Tabela 4). Já os esportivos, citaram maior diversidade como: vara, caniço, molinete, carretilha, tarrafa (embora seu uso seja vedado ao pescador esportivo), iscas vivas e artificiais (Tabela 3). Esta variação no uso de aparelhos está relacionada com o

profissionais, tanto da represa Jundiaí 
tipo de ambiente explorado e as espéciesalvo de determinadas pescarias.

As redes de espera são colocadas ao entardecer, por volta das $18 \mathrm{~h}$, e retiradas ao amanhecer, entre as $7 \mathrm{hs}$ e $8 \mathrm{hs}$ aproximadamente. O deslocamento para retirar ou colocar as redes é feito com o auxílio de barcos a motor ou barcos a remo somente quando as redes estão próximas à margem da represa.

Tabela 3 - Equipamentos mais utilizados pelos pescadores esportivos na captura do pescado $(n=30)$.

\begin{tabular}{lcc}
\hline & Represa Jundiaí & Represa Taiaçupeba \\
\hline Vara & $75 \%$ & $7,15 \%$ \\
Caniço & $75 \%$ & $7,15 \%$ \\
Molinete & $18,75 \%$ & $92,9 \%$ \\
Carretilha & $18,75 \%$ & $92,9 \%$ \\
Tarrafa & $6,25 \%$ & - \\
Iscas vivas & $100 \%$ & $100 \%$ \\
Iscas & $37,5 \%$ & $14,3 \%$ \\
artificiais & & \\
\hline
\end{tabular}

Tanto na Represa Jundiaí e Taiaçupeba, pontos de pesca são definidos por locais de fácil acesso, próximos a margem, ou seja, não existem melhores locais para pescar, levando-se em consideração o pescado. Dois pontos de pesca, onde ocorreu um maior número dos pescadores, foi no canal de escoamento, que liga as duas represas, e um braço da represa Jundiaí, que dá acesso à rodovia. Os pescadores têm conhecimento da época do ano (verão e inverno) em que os peixes são frequentemente mais capturados. Assim, existem os peixes de inverno (Rhamdia quelen), os peixes de verão (Salminus hilarii, Cyphocharax modestus, Gymnotus carapo, T. rendalli, O. niloticus, Hoplosternum littorale, Symbranchus marmoratus e Hypostomus ancistroides) e os peixes de ano todo (Astyanax fasciatus, Hoplia malabaricus, Geophagus brasiliensis e C. carpio).

Os pescadores atribuem sucesso ou fracasso das atividades pesqueiras a algumas variações das condições ambientais, tais como chuva, hora do dia e por se tratar de represas, quando está mais cheia ou mais vazia, pela abertura e fechamento dos canais. Estes fatores são importantes na tomada de decisão como a escolha dos pontos de pesca, os métodos mais adequados, as espécies a serem capturadas, entre outras.

Tabela 4 - Equipamentos mais utilizados pelos pescadores profissionais na captura do pescado.

\begin{tabular}{|c|c|c|}
\hline & Represa Jundiaí & Represa Taiaçupeba \\
\hline Barco a remo & $100 \%$ & $50 \%$ \\
\hline $\begin{array}{l}\text { Barco com } \\
\text { motor }\end{array}$ & $100 \%$ & $50 \%$ \\
\hline $\begin{array}{l}\text { Rede de } \\
\text { espera }\end{array}$ & $100 \%$ & $100 \%$ \\
\hline
\end{tabular}

Foram identificadas um total de 13 espécies de peixes (Tabela 5). A. fasciatus, S. hilarii, H. malabaricus, C. modestus, $C$. 
carpio, Gymnotus carapo, G. brasiliensis, T. rendalli, O. niloticus, Hoplosternum littorale, H. ancistroides e Rhamdia quelen foram capturadas por pescadores profissionais, e as espécies A. fasciatus, $G$. brasiliensis, $T$. rendalli, $O$. niloticus e $S$. marmoratus foram pescadas por pescadores esportivos. Cabe destacar que a espécie S. marmoratus, não ocorreu na Represa Jundiaí talvez por apresentar pouquíssimas macrófitas aquáticas como aguapé (Eichornia crassipes) e a alface d'água (Pistia stratiotes), onde esta espécie se abriga em fendas ou cavidades e também se reproduz.

Tabela 5 - Espécies capturadas pelos pescadores esportivos e profissionais nas represas Jundiaí e Taiaçupeba segundo BUCKUP et al., 2007.

\begin{tabular}{lc}
\hline CLASSIFICAÇÃO & NOME \\
POPULAR \\
\hline & \\
CHACIFORMES & \\
Characidae & \\
$\quad$ Astyanax fasciatus (Cuvier, 1819) & Lambari \\
Salminus hilarii (Valenciènnes, 1849) & Tabarana \\
Erythrinidae & \\
Hoplias malabaricus (Bloch, 1794) & Traíra \\
Curimatidae & \\
$\quad$ Cyphocharax modestus (Fernandez- & Saguirú \\
Yepes, 1948) & \\
CYPRINIFORMES & \\
Cyprinidae & \\
Cyprinus carpio (Linnaeus, 1758) & Carpa \\
GYMNOTIFORMES & \\
Gymnotidae & \\
Gymnotus carapo (Linnaeus, 1758) & Tuvira \\
PERCIFORMES & \\
Cichlidae & \\
Geophagus brasiliensis (Quoy \& & Cará \\
Gaimard, 1824) & \\
Tilapia rendalli (Boulenger, 1897) & Tilápia \\
Oreochromis niloticus (Linnaeus, \\
1758)
\end{tabular}

\section{DISCUSSÃO}

Informações disponíveis sobre a pesca no Brasil, geralmente são incompletas, obtidas por meio de várias metodologias que, algumas vezes, não têm valor científico (AGOSTINHO et al., 2007). Em reservatórios brasileiros, essa escassez de dados é originária, tanto da cultura do não-monitoramento, quanto de erros na obtenção de recursos e esforços que são desviados dessa atividade para ações como controle de pesca, estocagem, entre outras (AGOSTINHO et al., 2007). Esta situação tem dificultado uma avaliação da situação real dos recursos pesqueiros e a obtenção de um diagnóstico mais preciso da pesca, que acaba comprometendo o planejamento $\mathrm{e}$ as decisões sobre medidas de manejo (AGOSTINHO et al., 2007). 
O conjunto de conhecimentos que as comunidades pesqueiras locais apresentam sobre o comportamento, hábitos alimentares, reprodução e ecologia dos peixes, é uma importante fonte de informação, que pode auxiliar na definição de medidas de manejo da pesca e conservação da diversidade biológica (COSTA-NETO e MARQUES, 2000).

Em rios e represas do Estado de São Paulo, o monitoramento estatístico é ainda incipiente, porque não há uma cobertura satisfatória, as características da pesca artesanal continental são difusas e existe uma carência de pessoal técnico e de financiamento. No entanto, algumas concessionárias e o Instituto de Pesca realizam um monitoramento parcial (TORLONI et al., 1993; CESP, 1998; AGOSTINHO et al., 2007).

As espécies $C$. carpio, $T$. rendalli $\mathrm{e}$ $O$. niloticus são espécies exóticas, com alto valor comercial, introduzidas com a intenção de beneficiar a atividade pesqueira. Apresentam ótima aceitação no mercado por ser uma carne muito apreciada pelos consumidores; as ovas da carpa (C. carpio) também têm boa procura, atingindo um alto valor comercial, chegando a ser maior que o próprio pescado. Para AGOSTINHO e JULIO (1996), essa prática, salvo poucas exceções, não foi bem sucedida no sentido de provocar uma auto-sustentação da pesca, pois algumas espécies trouxeram graves consequências às comunidades de peixes nativos porque são espécies oportunistas, de rápida reprodução (4 vezes ao ano) e de hábito alimentar generalista, ou seja, fácil adaptação em ambientes lênticos (SMITH, 2003).

Todos os pescadores esportivos entrevistados afirmaram, como principal característica, o tamanho (comprimento) dos peixes para diferenciar os jovens dos adultos e que a captura de peixes jovens poderá levar ao desaparecimento da espécie naquela região. Como medida mitigatória, seria importante e necessário que os órgãos responsáveis pelas represas do Alto Tietê, o DAEE e a Sabesp, levassem informações sobre o manejo adequado e idade ideal da pesca de cada espécie de peixe presente nas represas, conscientizando aqueles que continuam a capturar indivíduos jovens das espécies nativas.

Em relação ao turismo, para os pescadores profissionais, a sujeira deixada pelos turistas, que utilizam a represa como recreação e natação, os pescadores amadores e a grande quantidade de pessoas que frequentam o local nos finais de semana, são os fatores que mais 
atrapalham e incomodam esses profissionais.

Um outro fator mencionado, que não está relacionado com o turismo, foi o efeito negativo do despejo de um algicida (sulfato de cobre) na Represa Jundiaí, por funcionários da Sabesp, com a finalidade de controlar o crescimento de macrófitas aquáticas flutuantes como aguapé ( $E$. cranipes) e a alface d'água ( $P$. stratiotes),

\section{AGRADECIMENTOS}

Os autores agradecem ao Prof. Dr. Miguel Petrere Jr. pela leitura criteriosa do

\section{REFERÊNCIAS BIBLIOGRÁFICAS}

AGOSTINHO, A. A.; JÚliO JR., H. F.; BORGHETTI, J. R. Considerações sobre os impactos dos represamentos na ictiofauna e medidas para sua atenuação. Um estudo de caso: Reservatório de Itaipu. Revista UNIMAR, Maringá, 14 (Suplemento): 89-107, 1992

AGOSTINHO, A. A. Considerações sobre a atuação do setor elétrico na preservação da fauna aquática $e$ dos recursos pesqueiros. In: SEMINÁRIO SOBRE FAUNA AQUÁTICA E O SETOR ELÉTRICO BRASILEIRO. REUNIÕES TEMÁTICAS PREPARATÓRIAS. Caderno I Fundamentos, Rio de Janeiro, RJ COMASE/ELETROBRAS. p.38-59, 1994.

AGOSTINHO, A. A. e JULIO JR., H. F. Peixes de outras áreas. Ciência Hoje, Rio de Janeiro, 21(124): 36-44, 1996. que pioram a potabilidade. Segundo os pescadores, esse algicida seria o responsável pela diminuição da população das espécies de peixes. Comprovou-se, nas observações de campo, que apenas uma espécie é prejudicada, a Platanichthys platana, que durante a coleta de dados, após a aplicação do sulfato de cobre, vários exemplares apareceram mortos em consequência do produto.

artigo e também pelas sugestões importantes para aprimorar o trabalho.

AGOSTINHO, A. A.; GOMES, L. C.; PELICICE, F. M. Ecologia e manejo de recursos pesqueiros em reservatórios do Brasil. Maringá. EDUEM, 2007. 501p.

BARRELLA, W. e PETRERE JR., M. Fish community alterations due to pollution and damming in Tietê and Paranapanema rivers (Brazil). River Research and Applications: 19: 59-76, 2003.

BAYLEY, P. B. e PETRERE JR., M. Amazon Fisheries: assessment methods, current status and management options. Canadian Suplement Publish Fish Aquatic Science., v. 106, p. 385-398. 1989.

BRITSKI, H. A. Seminário Sobre Fauna Aquática e o setor Elétrico. Caderno 1 Fundamentos. Foz do Iguaçu, PR, COMASE /ELETROBRAS, 1994. 55p. 
BUCKUP, P.A., MENEZES, N.A. \& GHAZZI, M.S. (eds.). Catálogo das espécies de peixes de água doce do Brasil. Museu Nacional, Rio de Janeiro, 2007, $195 \mathrm{p}$.

CASTRO, P. M. G.; CAMPOS, E. C.; SPIGOLON, J. R.; MARUYAMA, L. S. Diagnóstico da atividade pesqueira artesanal no Médio e Baixo Rio Tietê: uma análise crítica da situação atual. In: ENCONTRO BRASILEIRO DE ICTIOLOGIA, 15., São Paulo, 2003. São Paulo: Universidade Mackenzie/ Sociedade Brasileira de Ictiologia. 1 CD-ROM. 2003.

CASTRO, P. M. G.; MARUYAMA, L. S.; BEZERRA DE MENEZES, L. C.; MERCANTE, C. T J. Perspectiva da atividade pesqueira no Alto Tietê: contribuição à gestão dos usos múltiplos da água. Boletim do Instituto de Pesca, São Paulo, 32(1): 1-14. 2006.

CESP - Companhia Energética de São Paulo. Conservação e manejo nos reservatórios: Limnologia, ictiologia e pesca. CESP. São Paulo. Série Divulgação e Informação, 1998. 166p.

CETRA, M. e PETRERE Jr. M. Small scate fisheries in the middle River Taantins, Imperatriz (MA), Brasil. Fisheries Management and Ecology, Singapore, 8: 153-152. 2001.

COSTA-NETO, E. M. e MARQUES, J. G. W. A Etnotaxonomia de Recursos Ictiofaunísticos pelos Pescadores da Comunidade de Siribinha, Norte do Estado da Bahia, Brasil. Biociências, Porto Alegre, 8(2): 61-76. 2000.

DELARIVA, R. L. Ecologia trófica da ictiofauna do rio Iguaçu, PR e efeitos decorrentes do represamento de Salto Caxias. Maringá. 2002. 110p. (Tese de Doutoramento. Universidade Estadual de Maringá, UEM). 2002.
DIEGUES, A.C. Diversidade Biológica e Culturas Tradicionais Litorâneas: $O$ Caso das Comunidades Caiçaras. São Paulo: NUPAUB-USP. 1988.

DIEGUES, A. C. Povos e Mares: Leituras em Sócio- Antropologia Marítima. São Paulo, NUPAUB-USP. 1995.

HARDOY, J.; MILTIN, D.; SATTERHWAILE, D. Sustainable development and cities. In: Jorge E. Hardoy, Diana Mitlin, David Satterthwaite. (Ed.). Environmental problems in third world cities. London: Earthscan Publ. p.171- 201. 1992.

MEGGERS, B. Amazônia: a ilusão de um paraíso. Rio de Janeiro, Civilização Brasileira, 1997. 207 p.

MINTE-VERA, C. V. e PETRERE JR., M. Artisanal fisheries in urban reservoirs: a case study from Brazil (Billings Reservoir, São Paulo Metropolitan Region. Fisheries Management and Ecology, Singapore, (7): 537-549. 2000.

PEREIRA, C. C. G. F.; SMITH, W. S.; ESPINDOLA, E. L. G. Alterações tróficas nas espécies de peixes em decorrência dos reservatórios em cascata do Médio e Baixo Tietê. In: RECURSOS HIDROENERGÉTICOS: USOS, IMPACTOS E PLANEJAMENTO INTEGRADO, 1., São Carlos, São Carlos: p. 29-42. 2002.

REDE DE ÁGUAS. São Paulo, Disponível em:

http://www.rededasaguas.org.br/nucleo/alt o_tiete.htm> Acesso em: 15 fev. 2006. 2002.

ROOSEVELT, C.; HOUSLEY, R. A; IMAZIO DA SILVEIRA, M.; MARANCA, S.; JOHNSON, R. "Eighth 
Millenium Pottery from a Prehistoric Shell Medden in the Brazilian Amazon". Science, Washington, D.C., (254): 16211624. 1991.

SANTOS, R. A.; CÂMARA, J. J. C.; CAMPOS, E. C.; VERMULM Jr. H.; GIAMAS, M. T. D. Considerações sobre a pesca profissional e a produção pesqueira em águas continentais do Estado de São Paulo. Boletim Técnico Instituto de Pesca, São Paulo, (19): 1-32. 1995.

SILVANO, R. A. M. Ecologia de Três Comunidades de Pescadores do Rio Piracicaba (SP). 180 p. (Dissertação de mestrado. Instituto de Biologia, UNICAMP). 1997.

\section{SISTEMA DE PRODUÇÃO DO ALTO} TIETÊ. Disponível em http:// www.mananciais.org.br/site/mananciais_r msp/altotiete > Acesso em: 23 abr. 2006. 1994.

SMITH, W. S. \& W. BARRELLA. The Ichthyofauna of the marginal lagoons, SP, Brazil: Composition, Abundance and Effect of the Anthropogenic Actions. Revista Brasileira Biologia 52(4): 627-640. 2000.

SMITH, W. S. Os peixes do rio Sorocaba: A história de uma bacia hidrográfica. Grupo Artz. - Sorocaba, EDITORA TCM - Comunicações, 2003. 160p.

SMITH, W. S. A pesca no rio Tietê. Ciência Hoje, Rio de Janeiro, (38)223: 2027. 2006.

TORLONI, C. E. C.; CORRÊA, A. R. A.; CARVALHO, JR. A. A. D.; SANTOS, J. J. D.; GONÇALVES, J. L.; GERETO, E. J.; CRUZ, J. A.; MOREIRA, J. A.; SILVA, D. C.; DEUS, E. F.; FERREIRA, A. S. Produção pesqueira e composição das capturas em reservatório sob concessão da
CESP nos rios Tietê, Praná e Grande, no período de 1986 a 1991. CESP, Série Produção Pesqueira, São Paulo, 001: 73p. 1993.

TRIOLA, M. F. Introdução à Estatística. $9^{\circ}$ ed. Rio de Janeiro: LTC Editora, 2005. 653 p.

VIO, A. P. de A. Conservação da Fauna Aquática em Reservatórios. In: SEMINÁRIO SOBRE FAUNA AQUÁTICA E O SETOR ELÉTRICO BRASILEIRO, 3., Rio de Janeiro, Curitiba, Anais... Rio de Janeiro: ELETROBRÁS. p.24-29. 1994. 